\title{
Effects of Interfacial Layer on Refractive Index and Propagation of Waves in Small Spherical Metal/ Dielectric Composite
}

\author{
Dessalegn Kenate ${ }^{1, *}$, Sisay Shewamare ${ }^{2}$, Getnet Melese ${ }^{3}$ \\ ${ }^{1}$ Department of Physics, Jimma University, Jimma, Ethiopia \\ ${ }^{2}$ Department of Physics, Wolkite University, Wolkite, Ethiopia \\ ${ }^{3}$ Department of Physics, Debre Tabor University, Debre Tabor, Ethiopia
}

Copyright $\bigcirc 2018$ by authors, all rights reserved. Authors agree that this article remains permanently open access under the terms of the Creative Commons Attribution License 4.0 International License

\begin{abstract}
In this work we have studied the effect of interfacial layer on the refractive index, and propagation of waves in small spherical metal/dielectric composite separated by interfacial layers which is randomly embedded in a linear dielectric host matrix. The theoretical, and numerical descriptions are in terms of the interfacial factor (I) by incorporating Taylor expansion and Drude model. The result shows that both the interfacial layer property, and the percentage of the volume fraction of the metallic particles in the composite has an effect on the refractive index, and propagation of waves of the composite when the dielectric functions of the interfacial layer is more metal-like property than dielectric-like property.
\end{abstract}

Keywords Dielectric Function, Propagation of Waves, Optical Properties, Refractive Index, Interfacial Layer, Composites

\section{Introduction}

The nonlinear optical properties in disordered metal/dielectric composites have received much attention because of their potential application to optical correlated and phase conjugated device [1]. Many authors have studied the nonlinear optical response of such composite media $[1,2]$. In this paper we are interested to study the effects of interfacial layers on the refractive index, and propagation of waves in small spherical metal/dielectric composite separated by interfacial layer which is embedded in a linear dielectric host. The contribution of the interfacial layer on the linear and nonlinear response of the medium strongly effects on the propagation of electromagnetic wave, and refractive index in the optical material and can even result in the permanent modification of its physical properties [3].

\section{Effects of Interfacial Layer on Refractive Index of Small Spherical Metal in a Linear Host Matrix}

In the electrostatic approximation when the wavelength of the incident electromagnetic radiation is much greater than the typical size of the inclusion, the distribution of the potential in small spherical metal which is embedded in a linear dielectric host can be written as follows[4]:

$$
\begin{gathered}
\phi_{m}=-E_{a p p} A r \cos \theta, r \leq r_{1} \\
\phi_{i}=-E_{a p p}\left(B r-C r^{-2}\right) \cos \theta, r_{1} \leq \mathrm{r}<r_{1+t} 1 \\
\phi_{h}=-E_{a p p}\left(r-D r^{-2}\right) \cos \theta, r_{1+t}<r
\end{gathered}
$$

Where, $\boldsymbol{\phi}_{\boldsymbol{m}}, \boldsymbol{\phi}_{\boldsymbol{i}}$, and $\boldsymbol{\phi}_{\boldsymbol{h}}$ are potential of the metal, interfacial layer, and the linear dielectric host matrix respectively. $\boldsymbol{E}_{\boldsymbol{a p p}}$ is the applied filed, $\mathbf{r}$ and $\boldsymbol{\theta}$ are the spherical coordinates, $\boldsymbol{r}_{\mathbf{1}}, \boldsymbol{a n d} \boldsymbol{r}_{\boldsymbol{1 + \boldsymbol { t }}}$ are the radius of the metal and interfacial layer respectively. A, B, C and D are the unknown coefficients. Using the continuity conditions of the potential and displacement vector at the boundaries of metal-interfacial layer and interfacial layer-linear host matrix, we obtain an expression for the unknown coefficient's A, B, C, and D. The solution of the unknown coefficient's can be presented as

$$
\begin{aligned}
& \mathrm{A}=\frac{3 \varepsilon_{h}}{2 \varepsilon_{h}+\varepsilon_{m}+\frac{2 l}{r_{1}}}, \\
& \mathrm{~B}=\frac{2 \varepsilon_{h}}{2 \varepsilon_{h}+\varepsilon_{m}+\frac{2 I}{r_{1}}}, \\
& \mathrm{C}=\frac{-\varepsilon_{h}}{2 \varepsilon_{h}+\varepsilon_{m}+\frac{2 l}{r_{1}}} r_{1}^{3},
\end{aligned}
$$




$$
\mathrm{D}=\frac{\varepsilon_{m}+\frac{2 I}{r_{1}}-\varepsilon_{h}}{2 \varepsilon_{h}+\varepsilon_{m}+\frac{2 I}{r_{1}}}\left(r_{1+t}\right)^{3},
$$

I is the interfacial layer factor defined as $\mathrm{I}=\lim t \varepsilon_{S}$

$$
\mathrm{t} \rightarrow 0, \varepsilon_{s} \rightarrow \infty \text {. Where I is interfacial factor [5]. }
$$

Generally, for sharp and smooth interface denoted (i.e. I $=0$ ), there is no jump in the normal component of the electric displacement across the interface; whereas, for imperfect interface denoted by $I \neq 0$, the electric displacement jumps across the interface. We further remark that 'I' can be taken as positive or negative values, because the dielectric functions of metallic particles is made up of a large negative real and a small positive imaginary [6]. When I is taken as negative value, the interface exhibits metal-like behavior but for I positive value, the interface exhibits dielectric-like behavior.

The polarizability of the composite is

$$
\alpha=\frac{\varepsilon_{m}+\frac{2 I}{r_{1}}-\varepsilon_{h}}{2 \varepsilon_{h}+\varepsilon_{m}+\frac{2 I}{r_{1}}} .
$$

The real and imaginary parts of the polarizability of the composite are respectively given by:

$$
\begin{gathered}
\alpha^{\prime}=\frac{\left(\varepsilon_{m}^{\prime}+\frac{2 I}{r_{1}}-\varepsilon_{h}^{\prime}\right)\left(2 \varepsilon_{h}^{\prime}+\frac{2 I}{r_{1}}+\varepsilon_{m}^{\prime}\right)+\varepsilon_{m}^{\prime \prime 2}}{\left(2 \varepsilon_{h}^{\prime}+\frac{2 I}{r_{1}}+\varepsilon_{m}^{\prime}\right)^{2}+\varepsilon_{m}^{\prime \prime}} \\
\alpha^{\prime \prime}=\frac{3 \varepsilon_{m}^{\prime \prime} \varepsilon_{h}^{\prime}}{\left(2 \varepsilon_{h}^{\prime}+\frac{2 I}{r_{1}}+\varepsilon_{m}^{\prime}\right)^{2}+\varepsilon_{m}^{\prime \prime 2}}
\end{gathered}
$$

The refractive index is related with the dielectric function by the following relation

$$
n=\sqrt{\varepsilon}=\sqrt{1+4 \pi \alpha}
$$

Using the Clausius-Mossoti formula the effective dielectric functions of the composite can be written as:

$$
\frac{\varepsilon-\varepsilon_{h}}{\varepsilon+2 \varepsilon_{h}}=\frac{4 \pi}{3} D N
$$

Where $\mathrm{D}$ is given by equation (2d) and $\mathrm{N}$ is a density number of the inclusions. The volume fraction of spherical metallic particles in a linear dielectric host matrix

$$
f=\frac{4 \pi}{3} N\left(r_{1+t}\right)^{3}
$$

The dielectric function of the composite is expressed by:

$$
\varepsilon=\varepsilon_{h}[1+3 f \alpha]
$$

In these expressions we neglect the higher order because of $\boldsymbol{f} \boldsymbol{\alpha}<<1$. The real $\left(\boldsymbol{\varepsilon}_{1}\right)$ and imaginary $\left(\boldsymbol{\varepsilon}_{2}\right)$ parts of equation (9) are respectively described by the following expressions;

$$
\begin{gathered}
\varepsilon_{1}=\varepsilon_{h}^{\prime}+3 f \varepsilon_{h}^{\prime} \alpha^{\prime} \\
\varepsilon_{2}=3 f \varepsilon_{h}^{\prime} \alpha^{\prime \prime}
\end{gathered}
$$

By substituting the real parts of the polarizability in to the real parts of the dielectric function, and the imaginary parts of the polarizability in to the imaginary parts of the dielectric function and then by equating the real and imaginary parts of the refractive index $\boldsymbol{n}^{\prime}+\boldsymbol{i} \boldsymbol{n}^{\prime \prime}$ with the real and imaginary parts of the dielectric functions, we obtain a coupled equations for the real $n^{\prime}$ and imaginary $n^{\prime \prime}$ parts of the refractive index of the composite [1-6]

$$
\begin{gathered}
n^{\prime 2}-n^{\prime^{2}}=\varepsilon_{1} \\
2 n^{\prime} n^{\prime \prime}=\varepsilon_{2}
\end{gathered}
$$

The above equation yields the expression for the real $n^{\prime}$ and imaginary $n^{\prime \prime}$ parts of the refractive index of small spherical pure metal embedded in a linear dielectric host as:

$$
\begin{gathered}
n^{\prime}=\sqrt{\frac{1}{2}\left(\varepsilon_{1}+\sqrt{\varepsilon_{1}^{2}+\varepsilon_{2}^{2}}\right)} \\
n^{\prime \prime}=\sqrt{\frac{1}{2}\left(-\varepsilon_{1}+\sqrt{\varepsilon_{1}^{2}+\varepsilon_{2}^{2}}\right)}
\end{gathered}
$$

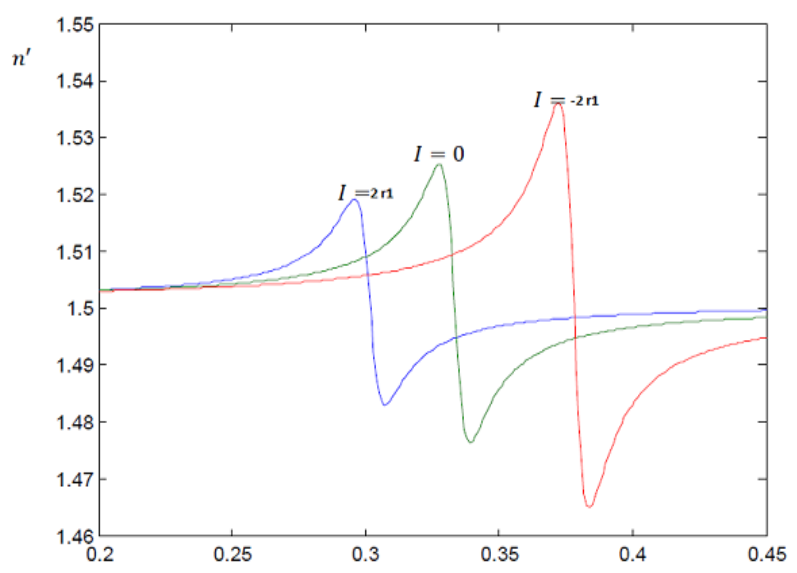

Figure 1. Refractive index $n^{\prime}$ of pure metal versus $\mathrm{z}$ for three different values of the interfacial layer factor, for the values of $\mathrm{f}=0.001$, and the numerical values of the composite parameters: $\varepsilon_{h}=2.25, \varepsilon \infty=4.5, \omega_{p}=$ $1.6 \times 10^{16} \mathrm{rad} / \mathrm{s}$, and $\gamma=0.0115$. For three diferent values of the interfacial layer we observethree diferent maximum value of therefractive index at different values of plasma resonance frequency. The resonance frquency of the composite is increased while, the corrospondingrefractive index is enhanced.

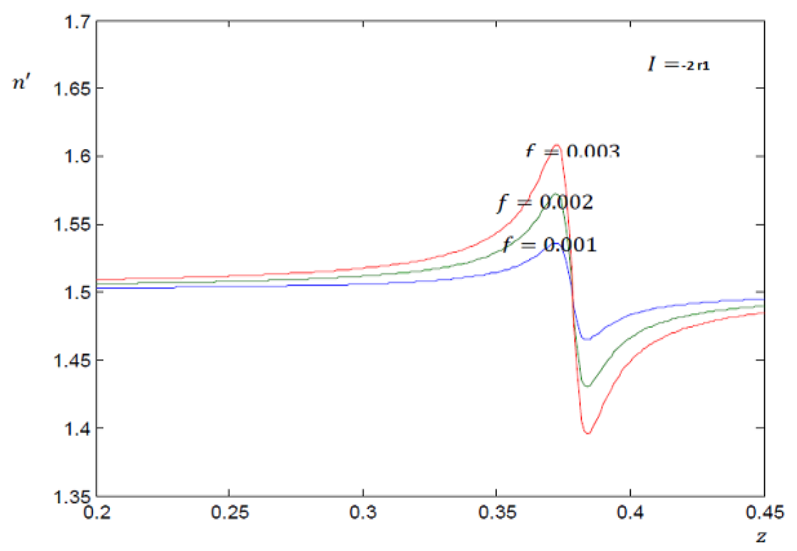

Figure 2. Refractive index $n$ of pure metal composite versus $\mathrm{z}$ for three different values of the percentage of metal fin linear host matrix; while the rest of the parameters are the same as in fig. 1 . 
In Figure 2 for three different values of the percentage of the volume fraction of the metal particles in the linear host matrix we observe different maximum values of the refractive index for a given value of the interfacial layer factor $\mathrm{I}=-2 r_{1}$. The increasing of the volume fraction of the metallic particles $\mathrm{f}$ in the linear host matrix increases the refractive index of the composite at the same resonance frequency.

\section{Effects of Interfacial Layer on Refractive Index of Small Spherical Metal/Dielectric Composite}

In the electrostatic approximation when the wavelength of the incident electromagnetic radiation is much greater than a typical size of the inclusion, the distribution of the electric potential of the composite system is described by the following expressions,

$$
\begin{gathered}
\phi_{d}=-E_{a p p} A r \cos \theta, r \leq r_{1} \\
\phi_{i}=-E_{a p p}\left(B r-C r^{-2}\right) \cos \theta, r_{1} \leq r_{1+t} \\
\phi_{m}=-E_{a p p}\left(D r-E r^{-2}\right) \cos \theta, r_{1+t} \leq r_{2} \\
\phi_{h}=-E_{\text {app }}\left(r-F r^{-2}\right) \cos \theta, r \geq r_{2}
\end{gathered}
$$

Where, $\phi_{d}, \phi_{i}, \phi_{m}$, and $\phi_{h}$ are potential of the dielectric, interfacial layer, metal, and the linear dielectric host matrix respectively. $\boldsymbol{E}_{\boldsymbol{a p p}}$ is the applied filed $\boldsymbol{r}$ and $\boldsymbol{\theta}$ are the spherical coordinates, $\boldsymbol{r}_{\mathbf{1}}, \boldsymbol{r}_{\mathbf{1 + t}}, \boldsymbol{a n d} \boldsymbol{r}_{\mathbf{2}}$, are the radius of the dielectrics, interfacial layer, and metal respectively. $\mathrm{A}, \mathrm{B}, \mathrm{C}, \mathrm{D}, \mathrm{E}$, and $\mathrm{F}$ are the unknown coefficients. Using the continuity conditions of the potential and displacement vector at the boundaries of dielectric-interfacial layer, interfacial layer-metal, and metal-linear host matrix, we obtain expression for the induced dipole moment of the polarization $\mathrm{F}$ as,

$$
F=\alpha r_{2}^{3}
$$

Where, the polarizability $\alpha=\left\{1-\frac{3 \varepsilon_{h\left[\left(\frac{3}{p}-1\right) \varepsilon_{m}+b\right]}}{2 \mathbf{h}}\right\}$, the denominator

$$
h=\varepsilon_{m}^{2}+K \varepsilon_{m}+b \varepsilon_{h}
$$

Where, $K=\left(\frac{3}{2 p}-1\right) b+\left(\frac{3}{p}-1\right) \varepsilon_{h}, p=1-\frac{r_{1}^{3}}{r_{2}^{3}}, \boldsymbol{b}=\varepsilon_{d}+\frac{2 I}{r_{1}}$.

The real $\alpha^{\prime}$ and imaginary $\alpha^{\prime \prime}$ parts of the polarizability respectively can be expressed in the form of

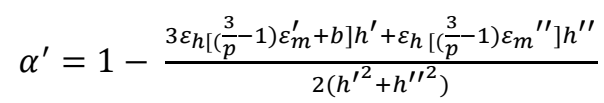

$$
\alpha^{\prime \prime}=\frac{\left.\left.3 \varepsilon_{h[}\left(\frac{3}{p}-1\right) \varepsilon_{m}^{\prime}+b\right] h^{\prime \prime}+\varepsilon_{h}\left[\frac{3}{p}-1\right) \varepsilon_{m}{ }^{\prime \prime}\right] h^{\prime}}{2\left(h^{\prime 2}+h^{\prime \prime}\right)}
$$

Also the real and the imaginary part of the denominator $\mathrm{h}$ is given in the following expressions

$$
\begin{gathered}
h^{\prime}=\varepsilon_{m}{ }^{2}+k \varepsilon_{m}^{\prime}+b \varepsilon_{h}-\varepsilon_{m}{ }^{\prime 2} \\
h^{\prime \prime}=\varepsilon_{m}^{\prime \prime}\left(k+2 \varepsilon_{m}^{\prime}\right.
\end{gathered}
$$

Substituting equation (15a) in to equation (10a) and equation (15b) into equation (10b), and then by equating the real and imaginary parts of the obtained expressions of the dielectric functions with the real and imaginary parts of the refractive index, we obtain the expressions for the real $\boldsymbol{n}^{\prime}$, and imaginary parts of the refractive index of metal/dielectric composite separated by interfacial layer as shown in equation (12).

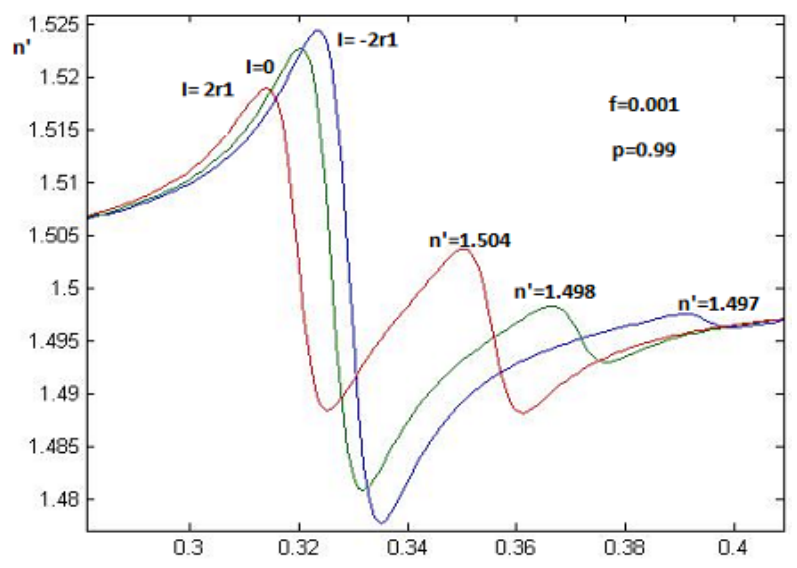

Figure 3. Refractive index $n^{\prime}$ of metal/dielectric composite verses z with interfacial layer factors $\mathrm{I}=-2 r_{1}, I=0, I=2 r_{1}$ for $\mathrm{f}=0.001$ and $\mathrm{p}=$ 0.99 , when $\varepsilon_{d}=6$. The rest of the parameters are the same as in fig (1).

From the above data we have seen three different maxima of refractive index of the composite at different plasma resonance frequency because of different value of the interfacial factor I. As the dielectric function of the interfacial layer $\varepsilon_{s}$ is changed from dielectric-like property to metal-like property the refractive index of the composite is enhanced, while the plasma resonance frequency is increased. But when the dielectric function of the interfacial layer $\varepsilon_{S}$ is changed from dielectric-like to metal-like property the refractive index of the composite is enhanced while, the plasma resonance frequency is decreased. If there is no interfacial layer effect on the refractive index of the composite, the peak of the refractive index of the composite is reduced.

This shows the interfacial layer plays a role on the refractive index of the composite. It is also noticed that such role is more important for metal-like interfacial layer than dielectric-like interfacial layer. 


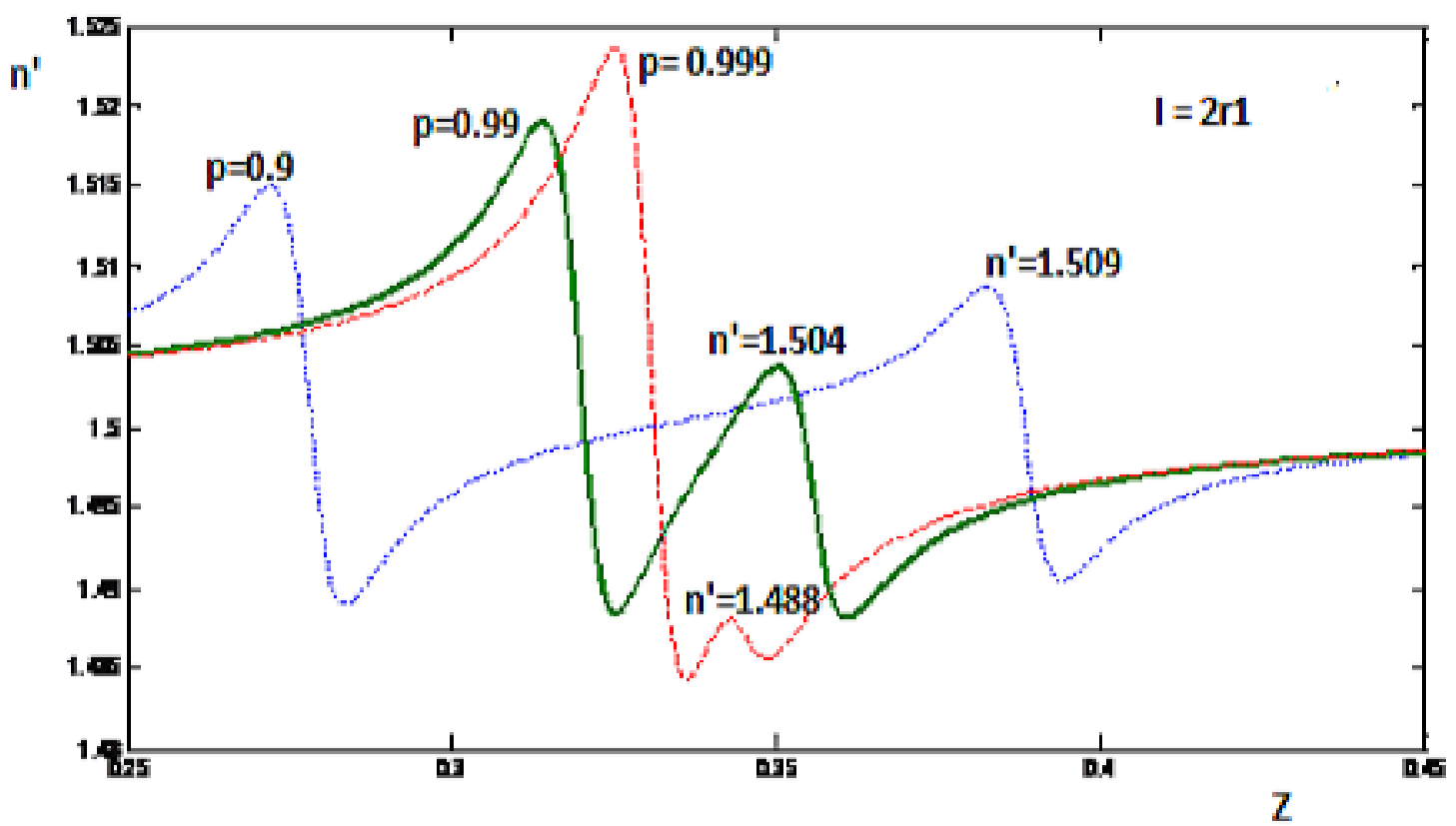

Figure 4. Refractive index $n^{\prime}$ of metal/dielectric composite verses $\mathrm{z}$ with interfacial layer factors $\mathrm{I}=2 r_{1}$ for three different values of metal fraction $\mathrm{p}$ $=0.9,0.99$, and 0.999 . When, $\boldsymbol{\varepsilon}_{\boldsymbol{d}}=\mathbf{6}$. The rest of the parameters are the same as in fig (1).

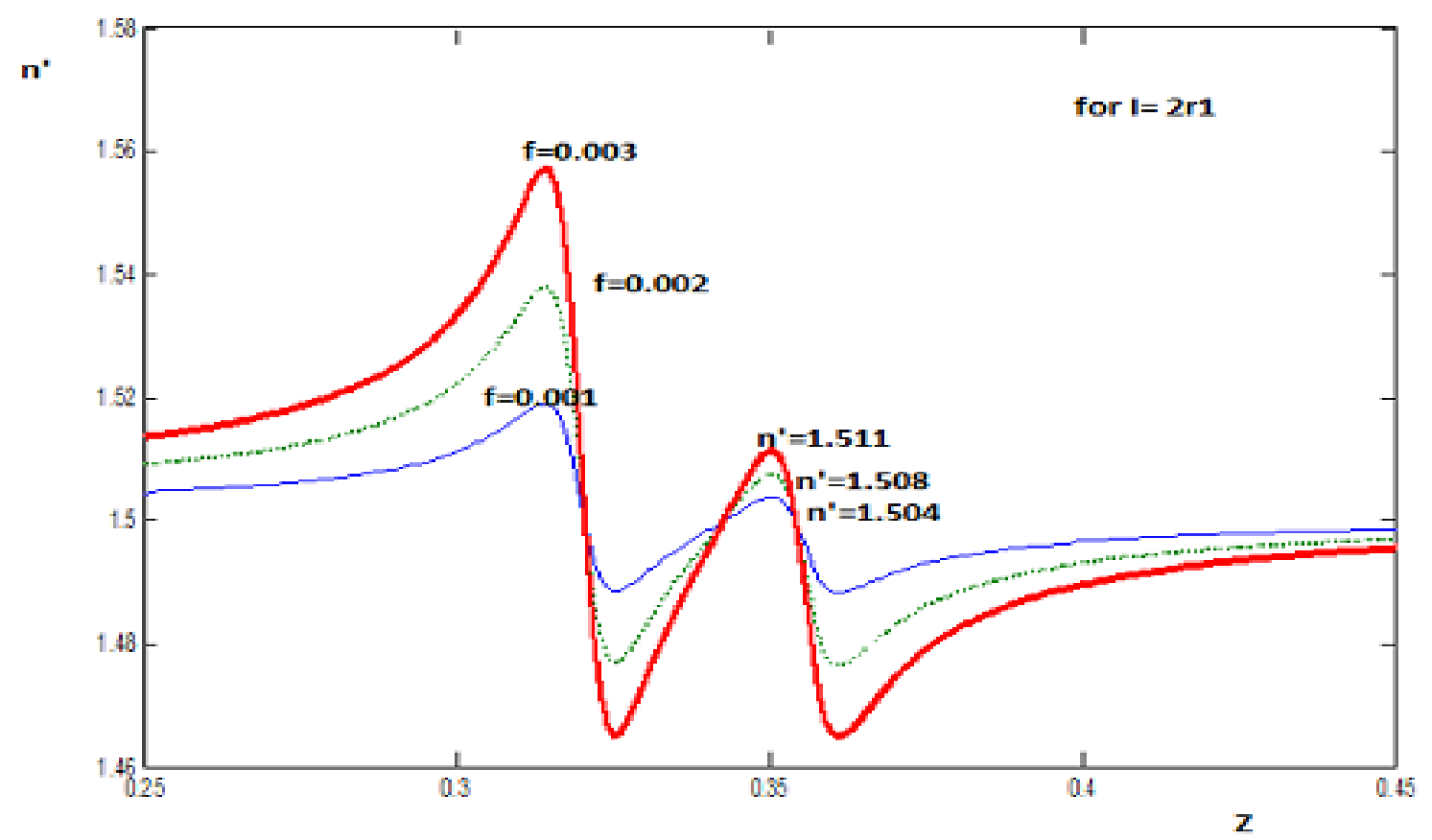

Figure 5. Refractive index $n^{\prime}$ of metal/dielectric composite verses $\mathrm{z}$ with interfacial layer factors $\mathrm{I}=2 r_{1}$ for three different values of percentage of particles $\mathrm{f}=0.001,0.002,0.003$. When $\varepsilon_{d}=6$. The rest of the parameters are the same as in fig (1).

In Figure (4) by keeping $\mathrm{I}=2 r_{1}$ and $\mathrm{f}=0.001$ we observe for the three different values of the metal fraction in the inclusion $p$, different values of the first, and second maxima of the real parts of the refractive index of the composite at different frequencies. For example for $p=0.9$ the first maxima is $n_{1}^{\prime}=1.515$ at frequency 0.272 , the second maxima is $n_{2}^{\prime}=1.509$ at frequency 0.383 , for $\mathrm{p}=$ 0.99 the $1^{\text {st }}$ and the $2^{\text {nd }}$ maxima is $n_{1}^{\prime}=1.519$ at frequency 0.314 , and $n_{2}^{\prime}=1.504$ at frequency 0.35 , respectively. And similarly for $\mathrm{p}=0.999$ the $1^{\text {st }}$ and $2^{\text {nd }}$ maxima is $n_{1}^{\prime}=1.524$ at frequency 0.325 , and $n_{2}^{\prime}=1.488$ at frequency 0.343 , respectively. Here the second maxima of the refractive index of the composite is decreasing and even for $\mathrm{I}=-2 r_{1}$ for the case of $\mathrm{p}=0.999$ second enhancement is very small.

Here we found that increasing the value of $f$ increases the refractive index of the composite. So for different values of, f we observe three different values of the $1^{\text {st }}$ maxima of the 
refractive index of the composite almost at the same resonance frequency, and the same is true for the $2^{\text {nd }}$ maxima. So the value of the parameter $f$ is not frequency dependent.

\section{Effects of Interfacial Layer on Propagation of Waves of Small Spherical Metal/Dielectric Composite}

The optical pulse propagating through highly dispersive $[7,8]$. Fig 6 . Depicts negative value of the group velocity $\left(v_{g}\right)$. In such consideration, the value of group velocity may be negative for two cases the $1^{\text {st }}$ prediction is when the peak of the transmitted pulse will exit the material before the peak of the incident pulse emerges the material, and in addition to that the $2^{\text {nd }}$ consideration is when the pulse will appear to propagate in the backward direction within the material [9]. We consider the propagation of the velocity of waves in the composite with the linear host matrix, $\varepsilon_{h}=0$. The group velocity can be calculated as [9]:

$$
\begin{gathered}
v_{g}=\frac{c}{n_{g}} \\
n_{g}(z)=n^{\prime}(z)+z \frac{\boldsymbol{d} \boldsymbol{n}^{\prime}}{\boldsymbol{d z}}
\end{gathered}
$$

which is called the group velocity of a wave packet. Where c is the speed of light in vacuum and $n_{g}$ is the group velocity index [9]. Let us consider the narrow wave packet centered at $k_{o}$ is given by the following equation $\boldsymbol{E}(\boldsymbol{t})=\int_{-\infty}^{\infty} \boldsymbol{e}^{-\boldsymbol{i}\left(\boldsymbol{k} x-\omega_{0} t\right)} \boldsymbol{e}^{\frac{-\left(\boldsymbol{k}-\boldsymbol{k}_{0}\right)^{2}}{2 \Delta^{2}}} d \boldsymbol{k}$. This relation is obtained from $v_{g}=\frac{d \omega}{d k}$, where, $\omega$ is the frequency and $k$ is the wave vector using the definition of the real part of the refractive index $n^{\prime}=\frac{k c}{\omega(k)}$, the group velocity $v_{g}$ appears in the second term of the Taylor series expansion,

$\omega_{k}=\omega_{k o}+v_{g}\left(k_{o}\right)\left(k-k_{o}\right)+\frac{1}{2} \frac{d v_{g}}{d k} / k=k_{o}\left(k-k_{o}\right)^{2}+$ ...

around the center of the wave packet $k_{o}$. Therefore, equation (14) has a meaning of the group velocity in the case of a week dispersion of $\omega(k)$. For a strong dispersion, the higher terms in equation (15) must be taken into account and in this case $V_{g}$, losses its physical meaning [9]. Finally, by substituting the real parts of the refractive index of small spherical pure metal/interfacial layer, and metal/dielectric composite into equation (14) we obtain the following numerical analysis respectively.

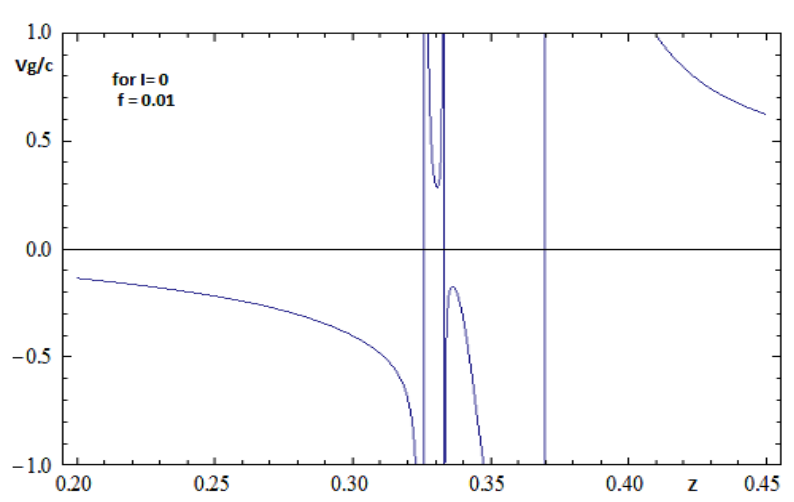

Figure 6. Normalized group velocity by speed of light $v_{g} / c$ versusz of the composite of pure metal/interfacial layer, when $\varepsilon_{h}^{\prime \prime}=0, f=$ $0.01, \varepsilon_{h}^{\prime}=2.25$, and $\varepsilon_{\infty}=4.5$

We observe that for the value of interfacial layer factor I $=0$, for constant $\mathrm{f}=0.01$. The negative group velocity $v_{g} / c<0$ is observed between frequency ranges of $(0.33$, $0.35)$ in this frequency range the negative group velocity or the propagation of light is rapidly increasing until it reaches the minimum of the group index $n_{g}$, which is the maximum point for the negative group velocity of the composite i.e. $v_{g}=-0.15 c$.

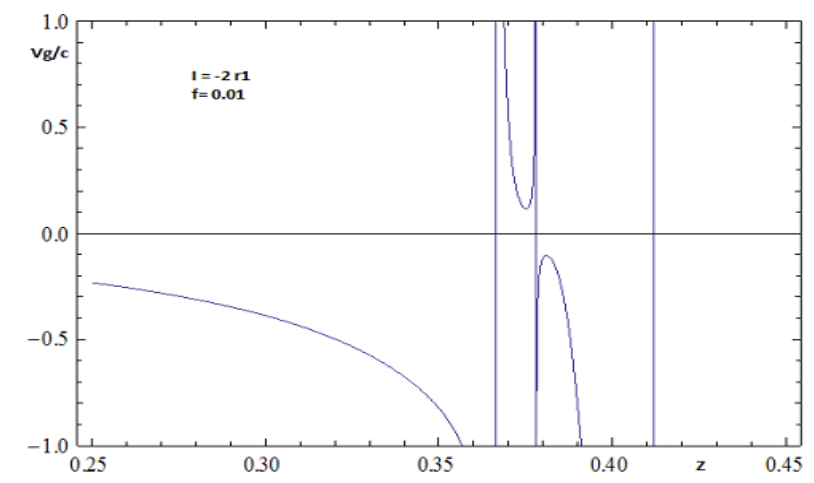

Figure 7. Normalized group velocity by speed of light $v_{g} / c$ versus $Z$ of the composite of pure metal with interfacial layer factor $I=-2 r_{1}$, when $\varepsilon_{h}^{\prime \prime}=0, f=0.01, \varepsilon_{h}^{\prime}=2.25$, and $\varepsilon_{\infty}=4.5$

In this figure we observe that for the value of interfacial layer factor $I=-2 r_{1}$, i.e. when the dielectric function of the interfacial layer is metal-like property, for constant $\mathrm{f}=0.01$. The superluminal light or the negative group velocity $\frac{V_{g}}{c}<0$ is observed between frequency ranges $(0.378,0.391)$ in this frequency range the negative group velocity or the propagation of light is rapidly increasing until it reaches the minimum of the group index $n_{g}$, which is the maximum point for the negative group velocity of the compositei.e $v_{g}=-0.13 c$. 


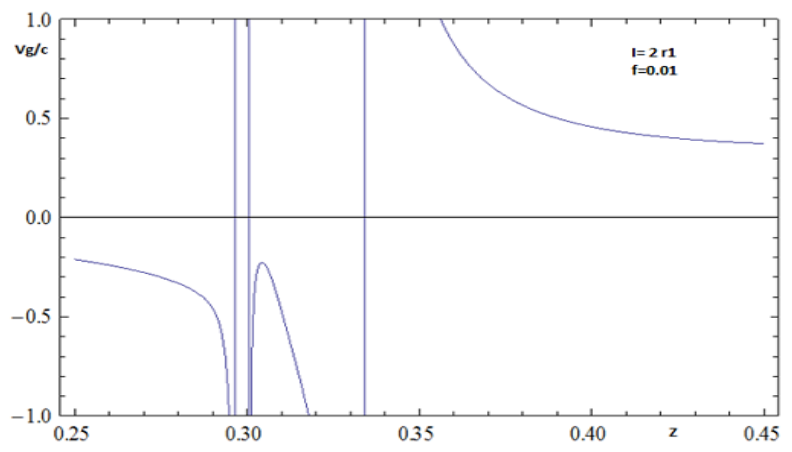

Figure 8. Normalized group velocity by speed of light $V_{g} / c$ versus $z$ of the composite of pure metal/interfacial layer factor, $I=2 r_{1}$, when $\varepsilon_{h}^{\prime \prime}=0, f=0.01, \varepsilon_{h}^{\prime}=2.25$, and $\varepsilon_{\infty}=4.5$.

In Figure-8, we observe that for the value of interfacial layer factor, $I=2 r_{1}$, i.e when the dielectric function of the interfacial layer factor is dielectric-like property, for a constant $\mathrm{f}=0.01$. The superluminal light (or negative group velocity) $\frac{v_{g}}{c}<0$ is observed between frequency rangesof $(0.30,0.319)$.In this frequency range the propagation of light is rapidly increasing until it reaches the minimum of the group index $n_{g}$ which is the minimum point for the negative group velocity of the composite i.e. $v_{g}=$ $-0.21 c$.

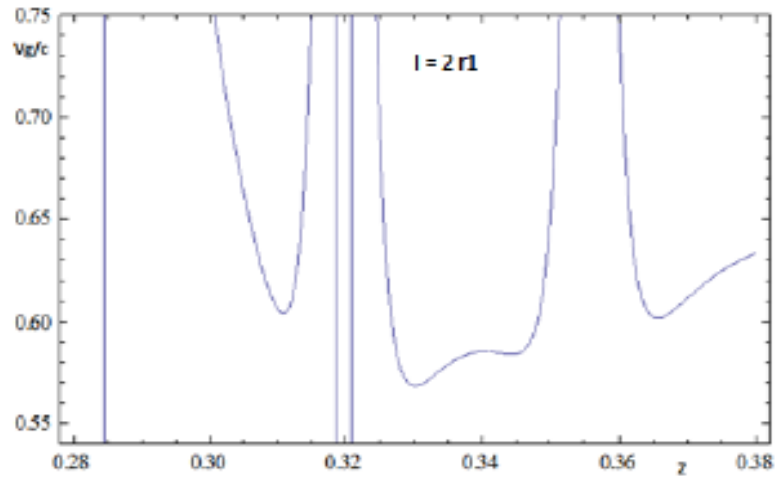

Figure 9. Normalized group velocity by speed of light $v_{g} / c$ versus $Z$ of the composite of small spherical metal/dielectric composite with interfacial layer factor $I=2 r_{1}, \mathrm{f}=0.01$, and $\mathrm{p}=0.99$.

In figure-9 we observe that for the value of interfacial layer factor $I=2 r 1$, i.e. when the dielectric function of the interfacial layer factor is dielectric-like property, for a constant $\mathrm{p}=0.99$, and $\mathrm{f}=0.01$. No superluminal light (or negative group velocity) $\frac{v_{g}}{c}<0$ is observed between frequency ranges $(0.25,0.40)$. In this frequency range the refractive index of the composite is minimum and also the value of absorption is less so that the group velocity is positive i.e. slow propagation of wave $v_{g} / c<1$.

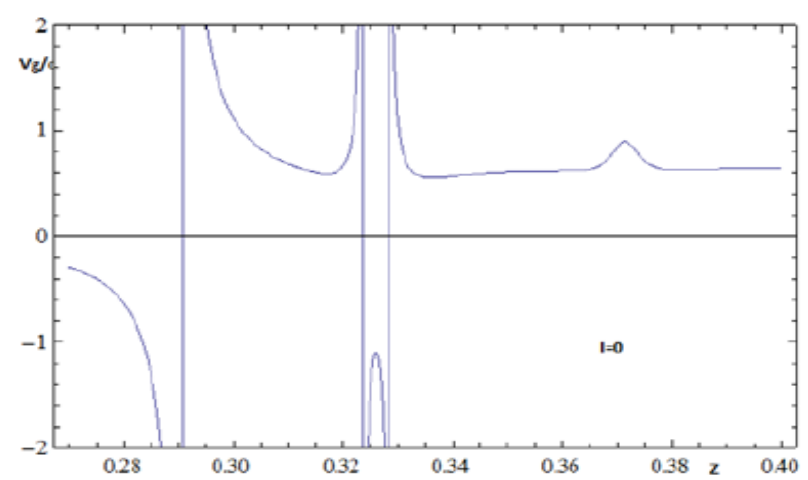

Figure 10. Normalized group velocity by speed of light $v_{g} / c$ versus $z$ of the composite of small spherical metal/dielectric composite with interfacial layer factor $I=0, \mathrm{f}=0.01$, and $\mathrm{p}=0.99$.

In this figure we observe that for the value of interfacial layer factor $\mathrm{I}=0, \mathrm{p}=0.99$, and $\mathrm{f}=0.01$. The superluminal light or the negative group velocity $\frac{v_{g}}{c}<0$ is observed between frequency ranges $(0.322,0.324)$ in this frequency range the group velocity or the propagation of light is rapidly increasing until it reaches the minimum of the group index $n_{g}$, which is the maximum point for the negative group velocity of the composite, i.e. $v_{g}=-1.18 \mathrm{c}$.

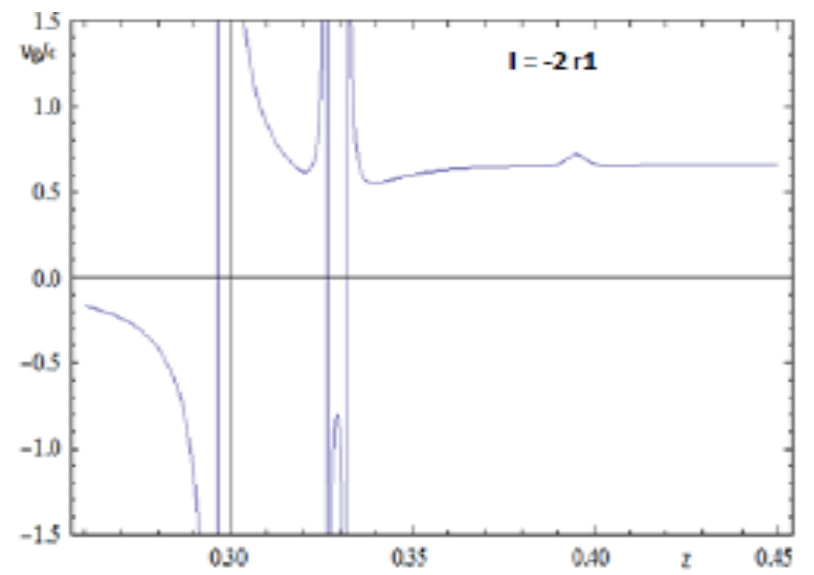

Figure 11. Normalized group velocity by speed of light $v_{g} / c$ versus z of the composite of small spherical metal/dielectric composite with interfacial layer factor $I=-2 r_{1}, \mathrm{f}=0.01$, and $\mathrm{p}=0.99$.

In figure-11 we observe that for the value of interfacial layer factor $\mathrm{I}=-2 r_{1}, \mathrm{p}=0.99$, and $\mathrm{f}=0.01$. The superluminal light or the negative group velocity $v_{g} / c<0$ is observed between frequency ranges $(0.325,0.335)$ in this frequency range the negative group velocity or the propagation of light is rapidly increasing until it reaches the minimum of the group velocity at the index $n_{g}$, which is the maximum point for the negative group velocity of the composite, i.e. $n_{g}=-0.75 \mathrm{c}$. 


\section{Conclusions}

The effects of the interfacial layer on the refractive index of the composite. When the dielectric function of the interfacial layer is changed from dielectric-like property to metal-like property the refractive index of the composite is more enhanced while, the frequency $\omega$ of the incident electromagnetic filed is increased. But when the dielectric functions of the interfacial layer is changed from metal-like property to dielectric-like property the refractive index of the composite is less enhanced while, the frequency $\omega$ of the incident electromagnetic filed is decreased. Maximum refractive index of the composite is obtained at less frequency of the incident electromagnetic filed when the dielectric functions of the interfacial layer is dielectric-like property but when the dielectric functions of the interfacial layer is metal-like property the maximum refractive index of the composite is obtained at high frequency of the incident electromagnetic filed. The refractive index of the composite is more enhanced when the dielectric function of the interfacial layer is metal-like property than dielectric-like property. At a given frequency of the incident electromagnetic filed the refractive index of the composite is more enhanced when the percentage of the volume fraction of the metallic particles in the inclusion is increased. We show the effects of the interfacial layer on the propagation of wave of the composite, for the case of pure metal with interfacial layer which is embedded in a linear dielectric host, for the value of $\mathrm{f}=0.01$ in comparison with no interfacial layer the maximum value of the negative group velocity is observed when the dielectric function $\left(\boldsymbol{\varepsilon}_{s}\right)$ of the interfacial layer is metal-like property than dielectric-like property.

In a small spherical metal/dielectric composite separated by interfacial layer which is embedded in a linear dielectric host, by decreasing the percentage of the volume fraction of metallic particles in the inclusion from 0.01 to 0.001 no negative group velocity is observed in the composite between frequency range (0.25-0.4) of incident electromagnetic filed, when the dielectric function of the interfacial layer is dielectric-like property but negative group velocity is found in the composite between frequency $(0.325-0.335)$ of the incident electromagnetic filed when the dielectric function of the interfacial layer is metal-like property. The Interfacial layer has an effect on the refractive index and propagation of waves in small spherical metal/dielectric composite separated by interfacial layer which is embedded in a linear dielectric host. It is also possible to control the negative group velocity of the composite by using the dielectric function $\boldsymbol{\varepsilon}_{\boldsymbol{s}}$ of the interfacial layer dielectric-like property.

\section{Acknowledgements}

Authors are grateful to Jimma University for financial support and the authors of the cited references.

\section{REFERENCES}

[1] Commun. Theor, Optical response of metal-dielectric composite containing interfacial layer, Physics, 36(2001), 251-256.

[2] V.N. Mal'nev, Sisayshewamare, Two optical bistability in composite of metal nanoparticles with nonlinear dielectric core, physca B 407(2012), 4837-4842.

[3] R.W. Boyd, Nonlinear Optics, Academic press, New York, 1992.

[4] T. pan, J.P. Huang, Z. Y. Li, phys, B 301(2001) 190.

[5] L, GAO and Z. Y. Li, J, Composite media, Appl, phys 87(2000), 1620.

[6] GAO. J. P. HUANG, L and Z. Y. Li, solid state commun, Appl. Phys, let. 115(2000), 347.

[7] E. M. Lifshitz L.D. Landau and L.P. Pitaevskii, Electrodynamics of continuous media, $2^{\text {nd }} \mathrm{ed}$, pergamon, oxford, 1984.

[8] Z. Dutton L. V. Han. S. E. Harris and C.H. Behoozi, Light speed reduction of 17 meters per second in an ultracold atomic gas, Nature 397(1999), 594-598.

[9] R.W. Boyd and D.J. Gauthie, "slow "and "fast" light in progress in optics 43(2002), 497-530. 